\title{
Nearest Neighbor Classification for High-Speed Big Data Streams using Spark
}

\author{
Swati T. Piske \\ M.S.Bidve Engineering \\ College, Latur
}

\author{
Tandle S. R. \\ Assistant Professor \\ M.S.Bidve Engineering \\ College, Latur
}

\begin{abstract}
High speed data streaming and data mining is the most contemporize challenges in machine learning. This demand methods displaying a high process effectiveness, with ability to continuously update their structure and handle ever-arriving big variety of instances. in this paper, we have a tendency to present a new incremental and distributed classifier based on the favored nearest neighbor algorithmic rule, adapted to such a exigent situation. This technique, enforced in Apache Spark, includes a distributed metric-space ordering to perform quicker searches. a vast live \{of information of data of knowledge containing useful data, referred to as big data, is created frequently. For handling such large volume of data, there's a necessity of big data structures, for example, Hadoop Map reduce, Apache Spark then on. Among these, Apache Spark performs up to one hundred circumstances speedier than ancient systems like Hadoop Map reduce. we have a tendency to concentrate on the plan of partition grouping calculation and its execution on Apache Spark.
\end{abstract}

\section{Keywords}

Nearest Neighbor, High-Speed Big Data,Data Streams

\section{INTRODUCTION}

A Huge live of information gets gathered ordinary because of the expanding inclusion of people within the computerized space. We share, store and deal with our work and lives on the web. as an example, Facebook stores more than thirty Petabytes of information, and Walmart's databases contain more than two.5 petabytes of knowledge. Such tremendous live of \{data of knowledge\} containing useful data is termed huge information. it's turning out to be more and more prevailing to mine such vast data keeping in mind the end goal to pick up bits \{of knowledge of data of information \} the important data that can be of incredible use in logical and business applications. Grouping is that the promisingdata miningprocedure that's generally embraced for mining vital data underlining unlabeled data.

A typical example, whereas all the closer neighbors are missing a minimum of one in all the query keywords, that the $\$ 64000$ nearest neighbor lies quite remote from the query purpose The introduction of web has given rise to associate degree ever increasing amount of text data related to multiple dimensions (attributes), for example client feed backs in on-line shopping web site like flip-kart as they are perpetually associated with the price, specifications and merchandise model. Keyword query, one in all the foremost popular and easy-to-use ways retrieves useful data from plain text documents. Given a group of keywords, existing ways aim to search out joins or all the relevant things that contains some or all the keywords. spacial queries with keywords has not been explored. Recently, attention was diverted to multimedia databases.

\section{HIGH SPEED BIG DATA ANALYSIS}

After obtaining neighbors the instance selector creates groups, where each one is formed by a new element and its neighbors. Then, local RNGE is applied on each group (as explained in Algorithm 3). The idea behind that is to build a local graph around each group and through this graph to decide what kind of action to perform on each element (insertion, removal, or none). New examples can be inserted or not, whereas old examples (neighbors) can be removed or maintained.

\subsection{Initial Partitioing Process}

The first step in the system consists of building a distributed metric tree formed by a top-tree called as the master machine and a set of local trees calledas the slave machines. This distributed tree will be queried and updated during next iterations with incoming batches of data. From the first batch we take a sample of $n t$ instances to build the main tree. The sampled data should be small enough to fit in a single machine and should maximize the separability between examples to avoid overlapping in the future subtrees. The $n t$ parameter is normally set to a value equal to the number of cores in the cluster. By doing so our algorithm is able to fully exploit the maximum level of parallelism in any stage.The routing tree is created following the standard procedure presented in where upper and lower bounds are defined to control the size of nodes.

Table 1 : Initial Partitioing Algorithm

\begin{tabular}{|c|}
\hline 1: INPUT: data, not \\
\hline 2: data is the input data-set \\
\hline 3: not Number of leaf trees to be distributed across the nodes \\
\hline 4: sample = smart Sampling(data) \\
using sample and the standard partitioning procedure \\
explained in It will be replicated to every slave machine. \\
\hline \begin{tabular}{c} 
6: For each leaf node in the top-Tree, one sub-tree is created \\
In a single slave machine. The resulting set of trees \\
(stored \\
As RDD) is partitioned and cached for further \\
processing \\
7: map-Reduce e $\in$ data \\
\hline 8: Find the nearest leaf node to e in top-Tree, and outputs a \\
tuple with the tree's ID (key) and e (value). (MAP)
\end{tabular} \\
\hline 9: The tuple is sent to the correspondent partition and \\
\hline
\end{tabular}


attached to the sub-tree according to its key. (SHUFFLE)

10: Combine all the elements with the same key (tree ID) by inserting them into the local tree. (REDUCE)

\section{1: Return the updated tree}

12: end map-Reduce.

\subsection{Updation Process}

When a new batch of data arrives, we need to start the updating process with edition. This is aimed at inserting new instances, as well as removing those that became redundant over time. At first, the algorithm computes which subtree eachelement falls into, following the same process described in theprevious section Once all instances are shuffled tosubtrees, a local nearest neighbor search for eachelement is started incorresponding subtrees.

Table 2 : Updation Process Algorithm

\begin{tabular}{|c|}
\hline 1: INPUT: query, ks, row \\
\hline 2: query is the data to be queried \\
\hline $\begin{array}{l}\text { 3: ksrepresents the number of neighbors to use in the instance } \\
\qquad \text { selection phase }\end{array}$ \\
\hline 4: row indicates whether to remove old noisy examples or not. \\
\hline 5: map-Reduce e $\in$ data \\
\hline $\begin{array}{l}\text { 6: Find the nearest leaf node to e in top-Tree and outputs a } \\
\text { tuple }\end{array}$ \\
\hline with the tree's ID (key) and e (value). (MAP) \\
\hline $\begin{array}{l}\text { 7: The tuple is sent to the correspondent sub-tree according to } \\
\qquad \text { its key. (SHUFFLE) }\end{array}$ \\
\hline $\begin{array}{l}\text { 8: neighbors = the standard M-tree search process is launched } \\
\text { for each element in its local sub-tree in order to retrieve the } \\
\text { ks-neighbors of e. The output will consist of a tuple with } \\
\text { e (key) and a list of its ks-neighbors (value). (REDUCE) }\end{array}$ \\
\hline $\begin{array}{c}\text { 9: edited = apply the local RNGE algorithm (Algorithm 3) to } \\
\text { each tuple in neighbors. The output consist of the } \\
\text { insertion/removal decision for each element }\end{array}$ \\
\hline 10: if row $==$ true then \\
\hline 11: Removed old noisy instances in edited from the tree. \\
\hline 12: end if \\
\hline 13: Add new correct instances in edited to the tree \\
\hline 14: Return the updated tree. \\
\hline 15: end map Reduce. \\
\hline
\end{tabular}

\subsection{Local RNGE}

After obtaining neighbors the instance selector creates groups, where each one is formed by a new element and its neighbors. Then, local RNGE is applied on each group (as explained in Algorithm 3). The idea behind that is to build a local graph around each group and through this graph to decide what kind of action to perform on each element

Table 3 : RNGE Algorithm

\begin{tabular}{|c|}
\hline 1: INPUT: e, net \\
\hline 2: incoming example \\
\hline 3: set of neighbors for e \\
4:Compute the local RNGE graph using e and net \\
following the procedure \\
\hline $\begin{array}{c}\text { 5: Mark e to be added iff most of its graph neighbors agree } \\
\text { with } \\
\text { its class }\end{array}$ \\
\hline 6: for en $\in$ net do \\
\hline 7:if en is a graph neighbor of e and most of en's graph \\
neighbors do not agree with its class then \\
\hline 8: Mark en to be removed \\
9: end if \\
\hline 10:end for. \\
\hline
\end{tabular}

\section{RESULT ANALYSIS}

The fallowing page (Fig. 1) is Home page. Basically it contains two tabs namely User Login and Admin login.

\subsection{Login Module}

This is the basic page of project module which contains as

User Login :Firstly u need to create a account by filling the basic required details.user name is the registered user email_id.For Login we need a email_id and passwordAnd if you are already a registered user the can go for Login

Admin Login :Admin site is nothing but server site on which different files are uploaded. An authorized user can access these files.

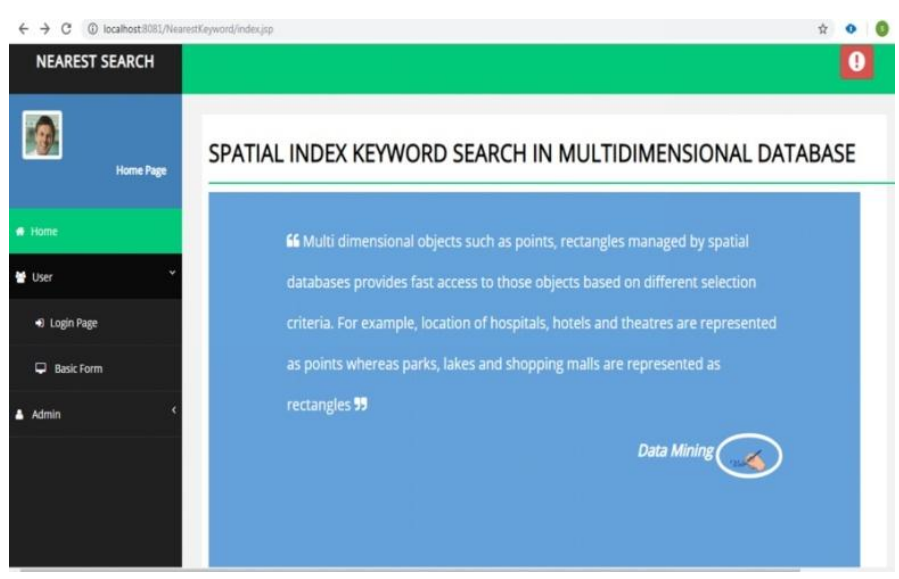

Fig 1: Home Page

\subsection{Client Sub-Modules :}

View Profile :The View profile contains basic information provided by user at the login time.

Request Secret key :As user is going to access data stored on server site for that it needs an authentication, for getting 
that the option / tab for search, user need to enter first an OTP called secret key send on users registered email id.Once user click on request key a key sent on id and if key match then tab is provided for keyword search.

View user-Search History :This module provide information about users set of keywords with users ID and date time when that user accessed data

View keywords \& related Data : This module contains information about keywords used by user while searching and even related data also displayed

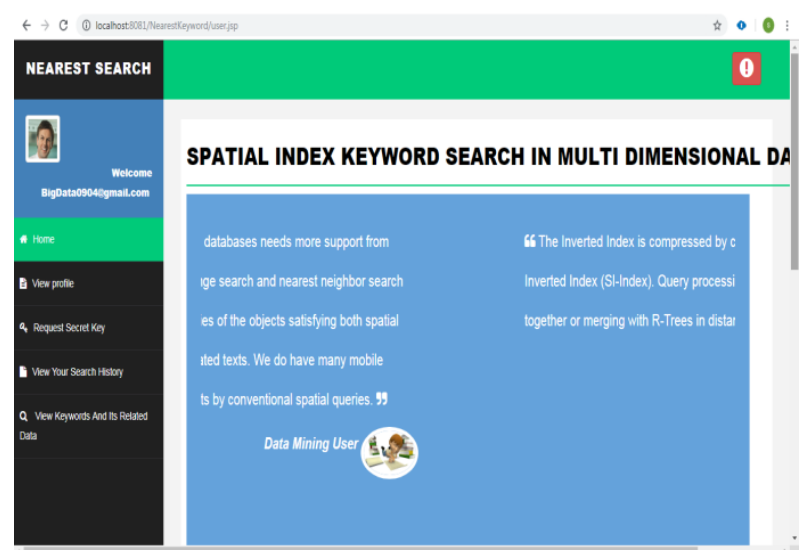

Fig 2: Client Sub-Module

\section{- Document Search :}

As user is going to search data stored on server using keywords there are two kind of files user can access, Document file and Text Image files. In document search user can search there types of files as :Text files,Doc files,Docx files,Simple image files

Here user need to enter only "text" as keyword to search required files, output is provided as different files related to keyword, the files may also be images.

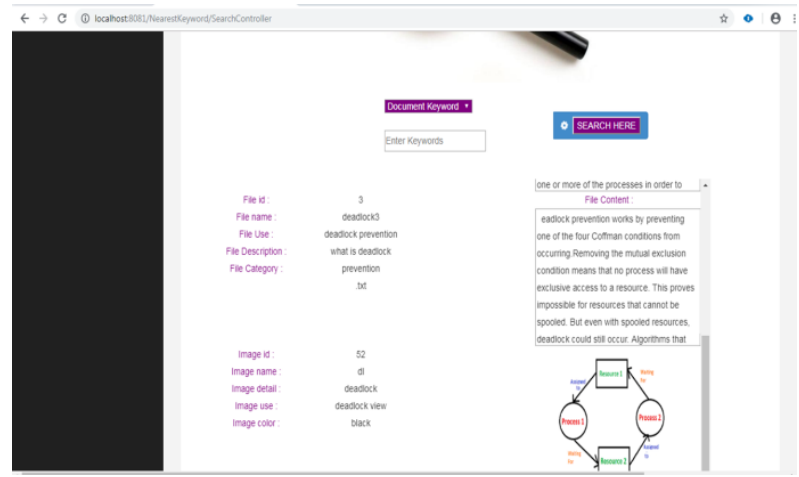

Fig 3: Document Search

\section{- TextImage Search :}

Then another file user can search are Text Image files means the images which has some text on it. Example: Suppose user want to search are Image files which contains "text" as "cloud" on it. Then userneed to select Text Image Search and enter keyword as cloud and result provide as image.

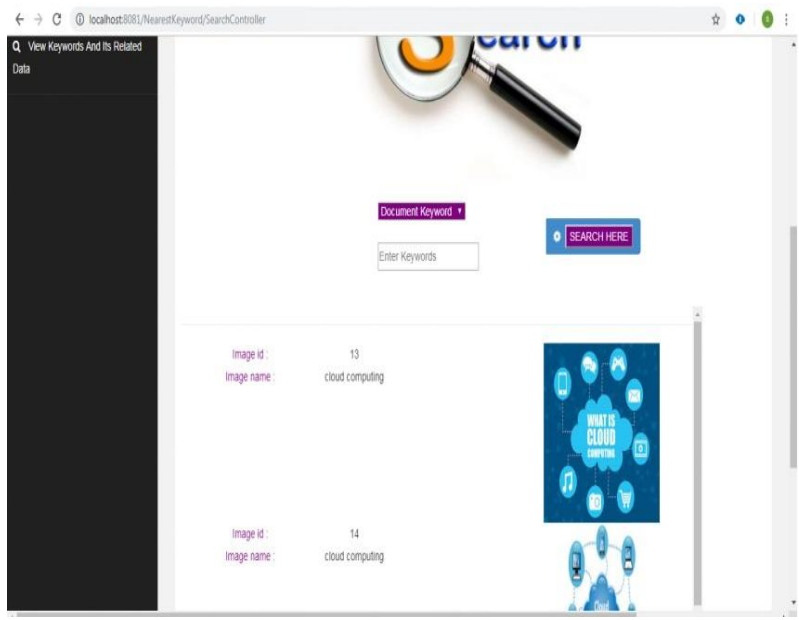

Fig 4: TextImage Search

\subsection{Admin Sub-Modules :}

On server site files it has different sub modules. It provide the options of uploading data and some features to view this uploaded data.

Add Document:This module provide feature of adding document files to server it requires name of file and extension.

Add Image: This module provides , adding the simple Image with proving basic dates.

Add Text image: This modules involves uploading Text images to server.

All Document: This module shows all those documents successfully uploaded and available on server

All simple images: This module shows all images which has some text on it.

All user: Number of user / clients registered can be viewed by all user module.

User search history: Admin can view what user searched for and even what keywords used by user with system date and time.

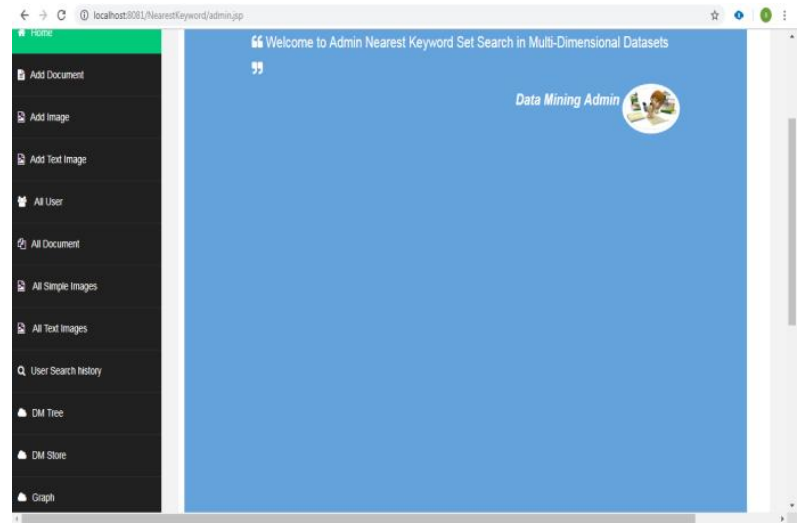

Fig 5 : Admin Sub-Modules

DM Tree : Data mining store provides keyword used for search and related files as name used DM Tree. So here root nodes and sub tree are considered.The root node $\mathrm{s}$ the keyword here and the leafs are nothing but files which contains keyword. Example: If user used keyword as "database" then it shows result "database". Keyword as "root" of tree and different file names which contains the keyword 
"database" in it as leaf nodes.

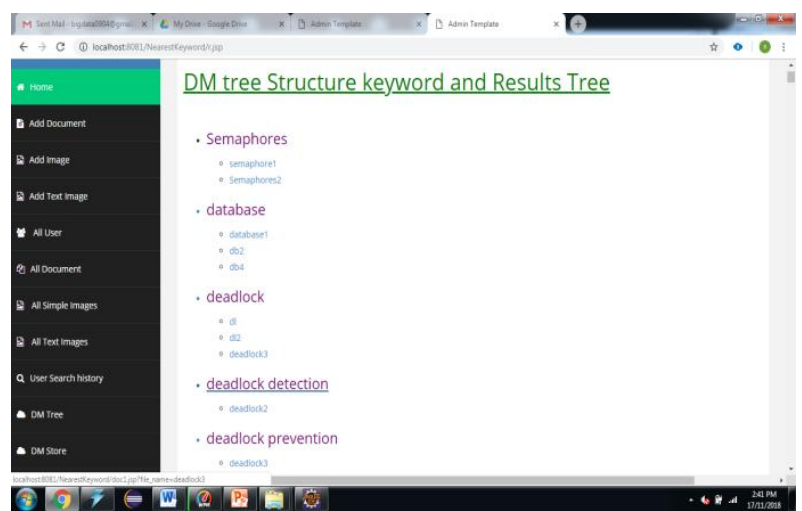

Fig 6 : DM Tree View

DM Store : Data mining store provides overall view of data. It shows the list of Document files, Image files and Text Image files uploaded on server site as:

Documents : The Document file names of all format

Images : All simple images uploaded.

TextImage : All TextImages uploaded to server

\subsection{Results}

The project work can only be seen by analyzing the results. Here inFallowing results aregenerated based on number of files keywords with search time of keyword.

The Lines with the blue showing graph for Document search and the line with black showing result for Image search.

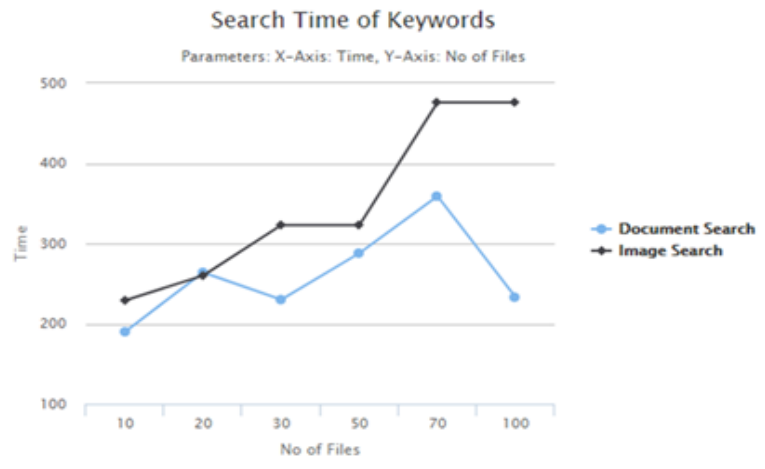

Fig 7 : First View of Ganerated Result

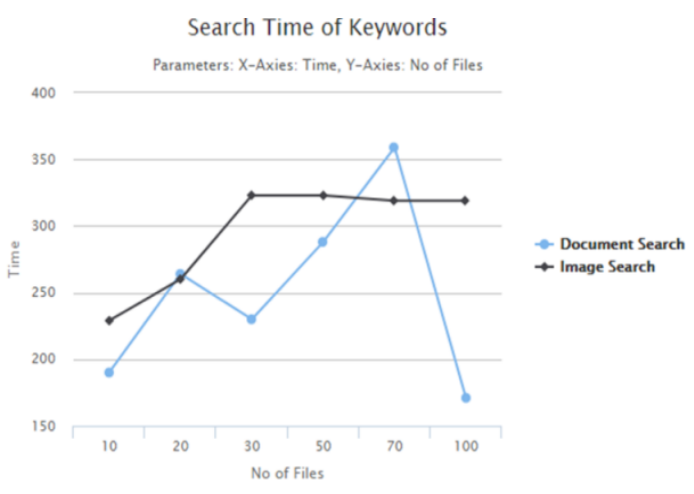

Fig 8 : Second View of Ganerated Result
We have presented DS-RNGE, a nearest neighbor classification solution for processing massive and high-speed data streams using Apache Spark. Up to our knowledge, DS-RNGE is the first lazy learning solution designed for large-scale, high-speed, and streaming problems. Our model organizes the instances by using a distributed metric tree, consisting of a top-level tree that routes the queries to the leaf nodes and a set of distributed sub-trees that performs the searches in parallel. DS-RNGE includes an instance selection technique that constantly improves the performance and effectiveness of the learner by only allowing the insertion of correct examples and removing outdated ones. As all phases in DS-RNGE perform the computations locally, our system is able to quickly respond to the continuous stream of data.

\section{ACKNOWLEDGMENTS}

This Apache Spark and Scala certification training is designed to advance your expertise working with the Big Data Hadoop Ecosystem. You will master essential skills of the Apache Spark open source framework and the Scala programming language, including Spark Streaming, Spark SQL, machine learning programming, GraphX programming and Shell Scripting Spark. This Scala Certification course will give you vital skillsets and a competitive advantage for an exciting career as a Hadoop Developer.

\section{REFERENCES}

[1] J. Gama, Knowledge Discovery From Data Streams. Boca Raton, FL, USA: Chapman \& Hall, 2010.

[2] Xindong Wu, Fellow, IEEE, XingquanZhu,’Data Mining with Big Data" IEEE Trans Big Data. vol. 26, no. 1, pp.97-107,Jan. 2014.

[3] N. Bharill and A. Tiwari, "Handling big data with fuzzy based classification approach," in Advance Trends in Soft Computing. Berlin, Germany: Springer, 2014, pp. 219-227.

[4] Bo Wu and HaiyingShen, Member, IEEE "Exploiting Efficient Densest Subgraph Discovering Methods"IEEE Trans Big Data,vol.3,pp.334-348,Sept.2017.

[5] Ming Shao, Member, IEEE, Xindong Wu, Fellow, IEEE, and Yun Fu, Senior Member, IEEE "Scalable Nearest Neighbor Sparse Graph Approximation by Exploiting Graph Structure" IEEE Trans Big Data.vol.2,pp.97- 107 Dec.2018.

[6] V. Mayer-Schönberger and K. Cukier, Big Data: A Revolution That Will Transform How We Live, Work and Think. London, U.K.: John Murray, 2013.

[7] D. Han, C. G. Giraud-Carrier, and S. Li, "Efficient mining of high-speed uncertain data streams," Appl. Intell., vol. 43, no. 4, pp. 773-785, 2015.

[8] U. Fayyad and R. Uthurusamy, "Evolving data into mining solutions for insights," Commun. ACM, vol. 45, no. 8, pp. 28-31, Aug. 2002. [Online]. Available: http://doi.acm.org/10.1145/545151.545174

[9] H. Karau, A. Konwinski, P. Wendell, and M. Zaharia, Learning Spark: Lightning-Fast Big Data Analytics. Sebastopol, CA, USA: O'Reilly Media, 2015.

[10] Apache Spark: Lightning-Fast Cluster Computing. (2017). Apache Spark. [Online].Accessed on Jan. 2017.[Online]. Available: https://spark.apache.org/. 